\title{
Transported to a World of Emotion
}

\author{
Simon Baron-Cohen*, Ofer Golan, Emma Chapman, Yael Granader
}

\section{INTRODUCTION}

Why would the UK government fund Cambridge University and a Manchesterbased production company to make a series of children's animation? After all, professional-quality animation doesn't come cheap. The answer is that this new animation has been designed to be both entertainment (for preschoolers) and educational: to help children learn to recognise emotions.

The government body in question is Culture Online, part of the Department of Culture, Media and Sport, set up five years ago. Their brief was to create innovative new applications of the new multimedia, with the specific mission of 'reducing exclusion' in society. Their commissioning executive, Claire Harcup, considered children with autism to be a group who remain excluded from society, because of their neurologically based difficulties in forming social relationships. Claire contacted us at the Autism Research Centre in Cambridge University to discuss a project.

We had already developed a DVD for teaching emotion-recognition to people aged eight and above on the autistic spectrum, called Mind Reading: The Interactive Guide to Emotion (1). Research has shown that using the DVD for two hours a week over a 10week period leads to significant improvement in emotion recognition among people with autism spectrum conditions (2). So our experience on that earlier project persuaded us that there are methods that can make a difference to people with this disability. Just as a child with dyslexia can be helped significantly by using tailored educational software to ease them into reading words, so a child with autism can be helped in the same way to understand emotions and read them from faces. Despite dyslexia being a form of word-

*To whom correspondence should be addressed:

Simon Baron-Cohen, Autism Research Centre, Department of Psychiatry, University of Cambridge.

E-mail: sb205@ cam. ac. uk. blindness, and autism being a form of mind-blindness (3), neither of these conditions is beyond remediation.

We took up the challenge offered by Culture Online by proposing to make an animated series called The Transporters. Whereas Mind Reading required children to be able to play a computer game, clicking a mouse, or to be supervised by a teacher or adult who could help them do this, The Transporters was aimed at relatively neglected ('excluded') people on the autistic spectrum: those with significant learning difficulties, and preschoolers. Neither of these two groups may have the ability or interest to use a computer, but both of these two groups enjoy watching animated films about vehicles.

So why do they love watching films about vehicles? According to one theory, children and adults with autism spectrum conditions are strong systemisers $(4,5)$. They are drawn to predictable, rule-based systems, whether these are repeating mathematical patterns, or repeating electrical patterns (e.g. light switches), or repeating patterns in films. They love lawful repetition.

It is ironic that Kanner, who first described autism in 1943, drew attention to this feature of autism (what he called their 'need for sameness' and their 'resistance to change') and yet it has been the social difficulties they have which have been the main focus of psychological research. At the core of autism is an ability to deal effortlessly with systems because they do not change and they are the same every time, and a disabling difficulty in dealing with the social world because it is always changing unpredictably and is different every time. It is worth noting that the two other major theories of autism that try to explain the non-social factors in autism - the weak central coherence theory (6) and the executive dysfunction theory (7) - have no parsimonious way of explaining this love of lawful repetition that is a hallmark of autism.

According to the hyper- systemizing theory (5), vehicles whose motion is determined only by physical rules (such as vehicles that can only go back and forth along linear tracks) would be much preferred by 
children with autism over vehicles like planes or cars, whose motion could be highly variable, moving at the whim of the human driver operating them. So we proposed to make a children's animation series based around eight characters who are all vehicles using such physical, rule based motion. Such vehicles would grab the attention of both preschoolers with autism and those so-called 'low functioning' children with autism with significant learning difficulties. Onto these vehicles we would graft real-life faces of actors, validated for the emotion that they were meant to be conveying, and contextualise them in entertaining social interactions between the toy vehicles.

Culture Online loved the idea and found a leading production company for us to work with. We created a whole family of different toy vehicles running on tracks or cables, who have limited freedom of motion: two trams, two cable cars, a chain ferry, a funicular railway, a coach and a tractor. Since all of the characters were depicted as toys in a child's bedroom, motion of the latter two was constrained in a Scalextric- like manner.

Each of the 15 episodes lasts five minutes, and opens with a sequence panning around the boy's bedroom where he plays with his toy vehicles. We then see the boy going off to school, and the vehicles 'coming to life', caught up in dramatic stories that enable the child watching to see different key emotions on the faces of the vehicles. The Transporters aims to teach not just some basic emotions (happy, sad, angry, disgust, fear, surprise) but also some more complex ones (ashamed, joking, jealous, proud, tired, sorry, kind, excited, worried, unfriendly and grumpy). Each short story is narrated (by actor Stephen Fry), but the series hopefully works even for a child without language, because the actions speak for themselves.

The DVD is widely and freely available (see box). The hope is that through hours of repetitive TV watching, children with autism will tune into faces without even realising they are doing so. Why? Because unlike faces on the people in their own homes, which are attached to human bodies that move unpredictably and are therefore stressful and confusing, the faces in The Transporters are attached to mechanical bodies that move beautifully predictably. The wheels turn. Round and round and round. The gears on the wheels lift, up and down and up and down. The vehicles move, back and forth and back and forth. Beautifully soothing for a child with autism who has a 'need for sameness'. Such systems, far from being confusing, are easy to understand because they are 100 per cent lawful, following the laws of mechanics, cause and effect. All you need in order to understand such mechanical motion are concepts like causality, temporal sequence, and contingency (If A, then B). And if you are a child who has difficulties with theory of mind or empathy, who finds it puzzling when a facial expression suddenly changes, the hope is that you could become familiar with how people look when they are surprised or afraid or proud through massive exposure to these patterns.

\section{DOES IT WORK?}

Our team has conducted an evaluation of The Transporters as an intervention. The study is under review with a peer- reviewed journal and will be made available in full via www.autismresearchcentre.com.

In the study, one group of 25 children with highfunctioning autism (aged four to seven years) were given copies of the animation series to use over a fourweek period, for 20 minutes a day. They were assessed prior to the intervention and at the end of it. A typically developing control group (matched on age, sex, IQ, handedness, language, and parental educational level) were simply assessed at two time-points with the same four- week interval in between. Results indicate that whilst the intervention group began at below- average levels on four tests of emotion-recognition, after intervention they achieved equivalent levels to the typically developing controls. (The tests included character's faces that had not appeared in the films themselves, thereby showing some degree of generalisation as well). This suggests that even with a relatively short intervention period, gains are possible.

The Autism Research Centre is planning further evaluations of The Transporters, to test if this is useful for different age groups as well as different subgroups on the autistic spectrum. As an intervention, it fits into a broader new approach for helping children with autism, which can be summarised by the phrase 'systemising empathy'. Empathy is usually acquired through nonsystemising means, but for children with autism this route may be a far more effective channel for developing empathy. Thus, Lego therapy $(8,9)$ is one example of how a system (Lego) can be used to motivate children with autism to socialise. For severe autism, it may be that no one single method is effective for all children. But researching such methods may enable us develop a range of methods that teachers, therapists and parents can turn to, to help such children connect with the social world.

\section{REFERENCES}

1. Baron-Cohen, S., Golan, O., Wheelwright, S. \& Hill, J.J.(2004). Mind reading: The interactive guide to emotions . London: Jessica Kingsley.

2. Golan, O. \& Baron-Cohen, S. (2006). Systemizing empathy: Teaching adults with Asperger syndrome or high functioning autism to recognize complex emotions using interactive multimedia. Development \&Psychopathology, 1 8, 589-615.

3. Baron-Cohen, S. (1995). Mindblindness:An essay on autism and theory of mind. MIT Press/Bradford Books. 
4. Baron-Cohen, S. (2002). The extreme male brain theory of autism. Trends in Cognitive Sciences, 6, 248- 254.

5. Baron-Cohen, S. (2006). Two new theories of autism:Hypersystemizing and assortative mating. Archives of Diseases in Childhood, 9 1, 2-5.

6. Frith, U. (1989). Autism: Explaining the enigma. Oxford: Blackwell.

7. Russell, J. (1994). Autism as an executive disorder. Oxford:Oxford University Press.
8. LeGoff, D.B. (2004). Use of LEGOC as a therapeutic medium for improving social competence. Journal of Autism and Developmental Disorders, 3 4(5), 557-571.

9. Owens, G., Humphrey, A. \& Baron-Cohen, S. (2006). LEGO ${ }^{8}$ therapy and the Social Use of Language Programme (SULP). Presentation at International Meeting for Autism Research conference.

Simon Baron-Cohen is Professor of Developmental Psychopathology at the University of Cambridge and Fellow at Trinity College, Cambridge. He is Director of the Autism Research Centre (ARC) in Cambridge (www.autismresearchcentre.com). He holds degrees in Human Sciences from New College, Oxford, a PhD in Psychology from UCL, and an M.Phil in Clinical Psychology at the Institute of Psychiatry. He held lectureships in both of these departments in London before moving to Cambridge in 1994.

He is author of Mindblindness (MIT Press, 1995), The Essential Difference (Penguin UK/Basic Books, 2003), and Prenatal Testosterone in Mind (MIT Press, 2005). He has edited a number of scholarly anthologies, including Understanding Other Minds (OUP, 1993, 2001), The Maladapted Mind (Erlbaum, 1997) and Synaesthesia (Blackwells, 1997). He has also written books for parents and teachers such as Autism and Asperger Syndrome: The Facts (OUP, 2008), and Teaching children with autism to mind read (Wiley, 1998). $\mathrm{He}$ is author of the DVD-ROM Mind Reading: an interactive guide to emotions (Jessica Kingsley Ltd, 2003) and The Transporters (www.thetransporters.com, 2007), an animation for preschool children with autism to help them learn emotion recognition. Both of these were nominated for BAFTA awards.

He has been awarded prizes from the American Psychological Association, the British Association for the Advancement of Science (BA), and the British Psychological Society (BPS) for his research into autism. For 2007 he was President of the Psychology Section of the BA, Vice President of the National Autistic Society, and received the 2006 Presidents' Award for Distinguished Contributions to Psychological Knowledge from the BPS. For 2009 he is Vice President of the International Society for Autism Research (INSAR). He is Patron of several autism and disability charities (Autism Anglia, Red2Blue, Autism Yorkshire, and Speaking Up). He is a Fellow of the BPS and co-editor in chief of the new journal Molecular Autism. His current research is testing the 'extreme male brain' theory of autism at the neural, endocrine and genetic levels. 